\section{ALIMENTAÇÃO NA ESCOLA E ESTADO NUTRICIONAL DE ESTUDANTES DO ENSINO FUNDAMENTAL}

\author{
Feeding in school and nutritional status of elementary school \\ students
}
Alimentación en la escuela y el estado nutricional de estudiantes de la enseñanza fundamental

Artigo Original

\section{RESUMO}

Objetivo: Relacionar o estado nutricional e o sexo de estudantes do ensino fundamental ao consumo da alimentação escolar gratuita, comprada nas cantinas e trazida de casa. Métodos: Estudo observacional, transversal, realizado em $2013 \mathrm{com} 120$ estudantes do $5^{\circ}$ ao $9^{\circ}$ ano de duas escolas municipais de ensino fundamental de um município gaúcho. Aplicou-se questionário estruturado para investigação acerca da alimentação escolar gratuita, comprada na escola ou trazida de casa. Foram aferidos peso, altura e circunferência da cintura (CC). Analisou-se a distribuição dos percentis do índice de massa corporal (IMC) e classificou-se o estado nutricional utilizando-se o Teste Exato de Fisher para análise. Resultados: Estudantes do sexo feminino ( $n=36 ; 46,8 \%)$ referiram não consumir a alimentação escolar gratuita porque não gostam, e os estudantes do sexo masculino $(n=22 ; 51,2 \%)$, porque não sentem fome $(\mathrm{p}=0,028)$. Com relação à alimentação comprada na escola, a preferência feminina $(n=12 ; 27,9 \%)$ foi por guloseimas, e a masculina $(n=21 ; 48,8 \%)$, por cachorro quente $(p=<0,001)$. A maioria dos estudantes classificados como eutróficos $(n=28 ; 33,3 \%)$ consumia a alimentação porque sentia fome, e os com sobrepeso/obesidade $(n=24 ; 70,6 \%)$, porque a considerava saudável e nutritiva $(\mathrm{p}=0,028)$. Conclusão: Os estudantes eutróficos, com sobrepeso e obesidade de ambos os sexos consomem de 2 a 3 refeições gratuitas oferecidas na escola por semana e demonstram preferência por trazer de casa bolachas, salgadinhos e sanduíches com pão branco. O sexo feminino prefere refrigerantes, guloseimas, doces e bolos, enquanto o sexo masculino, cachorro quente.

Descritores: Alimentação Escolar; Estado Nutricional; Estudantes.

\section{ABSTRACT}

Objective: To relate the nutritional status and sex of elementary students with the intake of free school meals, meals bought at canteens and meals brought from home. Methods: Cross-sectional observational study performed in 2013 with 120 students from 5th to 9th grade of two elementary and public school of a municipality in Rio Grande do Sul. A structured questionnaire was applied to investigate free school meals, meals purchased at school or brought from home. Weight, height and waist circumference (WC) were measured. The distribution of percentiles of Body Mass Index (BMI) was analyzed and the nutritional status was ranked, using the Fisher's Exact Test for analysis. Results: Female students $(n=36 ; 46.8 \%)$ reported not consuming the free school meals because they dislike $i$, and male students $(n=22 ; 51.2 \%)$ because they do not feel hungry $(p=0.028)$. Regarding the food purchased at school, females' preference $(n=12 ; 27.9 \%)$ was for candies and males' $(n=21 ; 48.8 \%)$ was for hot dog $(p=<0.001)$. The majority of the students classified as eutrophic $(n=28 ; 33.3 \%)$ consumed the food because they were hungry, and those classified as overweight/obese ( $n=24 ; 70.6 \%)$, because they considered it healthy and nutritious ( $p=0.028$ ). Conclusion: Most eutrophic, overweight and obese students in both genders consume 2-3 free meals offered at school per week and they give preference to bringing from home some biscuits, snacks and sandwiches made with white bread. Females prefer soft drinks, goodies, candies, and cakes, while males prefer the hot dog.

Descriptors: School Feeding; Nutritional Status; Students.

\author{
Jeani Tedeschi Ferreira ${ }^{(1)}$ \\ Joana Lemos ${ }^{(1)}$ \\ Simone Morelo Dal Bosco ${ }^{(1)}$ \\ Fernanda Scherer Adami ${ }^{(1)}$
}

1) Centro Universitário - Univates - Lajeado (RS) - Brasil

Recebido em: 06/12/2013

Revisado em: 03/03/2014 Aceito em: 24/04/2014 


\section{RESUMEN}

Objetivo: Relacionar el estado nutricional y el sexo de estudiantes de la enseñanza fundamental con el consumo de la alimentación gratuita de la escuela, la de las cafeterías de la escuela y las que se trae de casa. Métodos: Estudio observacional y transversal realizado en 2013 con 120 estudiantes del $5^{\circ}$ al $9^{\circ}$ año de dos escuelas municipales de enseñanza fundamental de un municipio del Sur de Brasil. Se aplicó un cuestionario estructurado para investigar sobre la alimentación gratuita de la escuela, aquella comprada en la cafetería o la que se trae de casa. Fueron verificados el peso, la altura y circunferencia de la cintura (CC). Se analizó la distribución de los percentiles del índice de masa corporal (IMC) y se clasificó el estado nutricional utilizándose la prueba Exacto de Fisher. Resultados: Estudiantes del sexo femenino $(n=36 ; 46,8 \%)$ refirieron que no consumian la alimentación gratuita de la escuela porque no les gustan y los estudiantes del sexo masculino ( $n=22 ; 51,2 \%)$ porque no tienen hambre $(p=0,028)$. Respecto la alimentación adquirida en la escuela la preferencia femenina ( $n=12 ; 27,9 \%)$ fue por golosinas y la masculina $(n=21 ; 48,8 \%)$ por perrito caliente $(p=<0,001)$. La mayoría de los estudiantes clasificados como eutróficos ( $n=28 ; 33,3 \%)$ consumian la alimentación porque tenían hambre $y$ aquellos con sobrepesolobesidad $(n=24 ; 70,6 \%)$ porque la consideraba saludable y nutritiva $(p=0,028)$. Conclusión: Los estudiantes eutróficos de ambos sexos con sobrepeso y obesidad consumen entre 2 y 3 comidas gratuitas ofrecidas en la escuela cada semana y demuestran la preferencia de traer de casa galletas, ganchitos y emparedados de pan blanco. El sexo femenino prefiere refrescos, golosinas, dulces y pasteles mientras el sexo masculino prefiere el perrito caliente.

Descriptores: Alimentación Escolar; Estado Nutricional; Estudiantes.

\section{INTRODUÇÃO}

A alimentação escolar chega gratuitamente até os alunos de escolas públicas e filantrópicas através do Programa Nacional de Alimentação Escolar (PNAE) e abrange toda a educação básica (educação infantil, ensino fundamental, ensino médio e educação de jovens e adultos). Seu objetivo é atender às necessidades nutricionais dos alunos durante sua permanência em sala de aula, contribuindo para o crescimento, desenvolvimento, aprendizagem e rendimento escolar dos estudantes, bem como promover a formação de hábitos alimentares saudáveis ${ }^{(1)}$. Daí a importância de saber se o programa funciona adequadamente, se os alimentos oferecidos são consumidos de forma adequada e se a alimentação está sendo bem aceita pelos alunos ${ }^{(2)}$. O gestor deve garantir o acesso universal, equânime, regular e contínuo à alimentação saudável; apoiar o desenvolvimento sustentável e o controle social; promover o resgate dos hábitos alimentares regionais; planejar e monitorar as ações de oferta da alimentação e as ações de educação alimentar e nutricional ${ }^{(3)}$.

Portanto, uma melhora da alimentação escolar é de extrema importância, sendo o acompanhamento nutricional uma ferramenta fundamental para a monitoração do estado de saúde dessa população ${ }^{(4)}$, que vive um período de transição nutricional no qual é visível o aumento dos índices de sobrepeso e obesidade e a diminuição da desnutrição infantil, influenciados por fatores sociais, ambientais, econômicos e principalmente de saúde ${ }^{(5,6)}$. A desnutrição, que era um fator de risco observado, hoje apresenta redução, mudando o problema da saúde pública para o sobrepeso e a obesidade. Os números aumentam todos os dias de forma muito rápida e difícil de ser contida, conhecendo-se que metade da população total e um terço das crianças de cinco a dez anos de idade estão em sobrepeso ou obesidade ${ }^{(7,8)}$.

A política pública da alimentação escolar é uma das estratégias desenvolvidas pelo Estado para minimizar problemas educacionais e sociais. Em um país como o Brasil, é possível observar desigualdade social e, por isso, não basta apenas oferecer uma escola pública de boa qualidade às crianças, é necessário também que elas estejam bem alimentadas, a fim de evitar o aumento de evasão e melhorar o rendimento escolar ${ }^{(9)}$.

Assim, identificar os motivos que levam o aluno a não consumir a refeição tanto em domicílio como na escola tornase fundamental para garantir a manutenção de sua qualidade de vida, já que a alimentação inadequada ou insuficiente acarreta problemas no desenvolvimento da criança e a consequente perda do rendimento escolar ${ }^{(10)}$. Na maioria das vezes, a preferência alimentar dos estudantes recai sobre alimentos não nutritivos e de alta densidade energética que são consumidos com frequência na escola ${ }^{(11)}$. Há um grande desafio em relação à modificação da realidade das cantinas escolares, por meio de ações de educação alimentar e nutricional associadas à redução da oferta de alimentos considerados não saudáveis ${ }^{(10)}$.

Baseado no exposto, o objetivo do presente estudo foi relacionar o estado nutricional e o sexo de estudantes do ensino fundamental ao consumo da alimentação escolar gratuita, comprada nas cantinas e trazida de casa.

\section{MÉTODOS}

Trata-se de estudo observacional, transversal, realizado entre agosto e outubro de 2013, o qual convidou 290 estudantes do $5^{\circ}$ ao $9^{\circ}$ ano, com idades entre 11 e 16 anos, todos matriculados em duas escolas municipais de ensino fundamental, atendidas pelo Programa Nacional de Alimentação Escolar (PNAE) do município de LajeadoRS. Dos 290 estudantes, 120 retornaram com o Termo de Consentimento Livre e Esclarecido (TCLE) assinado 
pelos pais ou responsáveis, sendo inseridos no estudo. As variáveis de idade e sexo foram obtidas por meio de pesquisa nos registros de documentos escolares.

Utilizou-se para a coleta de dados um questionário estruturado especialmente para a presente pesquisa, composto por quatorze questões objetivas, as quais investigavam: o tipo de alimentação escolar oferecida, os alimentos que os estudantes traziam de casa, a importância da alimentação para os estudantes, quantas vezes na semana era consumida a alimentação escolar oferecida gratuitamente, motivos pelo qual não era consumida, o que costumavam beber durante o período da alimentação escolar e o nível de aceitação desta. O questionário impresso era respondido pelos estudantes em uma sala destinada à atual pesquisa. Eram disponibilizados em torno de trinta minutos para respondê-lo.

Para a avaliação do estado nutricional, foram aferidos peso, altura e circunferência da cintura (CC) dos estudantes ${ }^{(12)}$. A partir do registro das medidas antropométricas, analisaram-se os percentis do índice de massa corporal (IMC), que foram classificados, conforme escore Z, nas curvas da Organização Mundial da Saúde $(\mathrm{OMS})^{(13)}$.

Aferiu-se, ainda, o peso, em balança portátil digital para adultos Plenna ${ }^{\circledR}$ (até $150 \mathrm{~kg}$ e divisão de $100 \mathrm{~g}$ ), com os estudantes posicionados no centro da balança, descalços e com roupas leves. Por meio de um estadiômetro portátil Wiso $^{\circledR}$, verificou-se a altura dos estudantes, estando estes descalços, com calcanhares juntos, costas retas e braços estendidos ao lado do corpo. A CC foi determinada por apenas um avaliador, com os estudantes em posição ereta $\mathrm{e}$ braços estendidos, utilizando-se uma fita métrica flexível e inelástica, com precisão de uma casa decimal e classificada conforme percentis para avaliação da $\mathrm{CC}(\mathrm{cm})$ em crianças e adolescentes ${ }^{(12)}$.

Os dados foram apresentados em números absolutos e percentuais. Para verificar a associação entre as variáveis de consumo (alimentação escolar gratuita, alimentação comprada nas cantinas, alimentação trazida de casa e líquidos consumidos), o estado nutricional e o sexo dos estudantes analisados, utilizou-se o Teste Exato de Fisher, com nível de significância máximo assumido de 5\% $(\mathrm{p}<0,05)$, através do software Statistical Package for the Social Sciences (SPSS), versão 13.0.

O estudo recebeu aprovação do Comitê de Ética em Pesquisa do Centro de Ciências da Saúde do Centro Universitário Univates, sob o número 335.696, em consonância com a Resolução 466/12 do Conselho Nacional de Saúde.

\section{RESULTADOS}

Dos 120 estudantes analisados, $77(64,2 \%)$ eram do sexo feminino, com idade média de 12,77 anos $( \pm 1,49)$. Segundo a classificação do IMC, 84 (70\%) encontravam-se eutróficos; 20 (16,7\%), com sobrepeso; e $14(11,7 \%)$, com obesidade. A classificação da CC teve adequação em 118 estudantes $(98,3 \%)$ (Tabela I).

Quarenta e seis $(n=46 ; 59,7 \%)$ estudantes do sexo feminino e $23(53,5 \%)$ do sexo masculino consideraram a alimentação escolar importante, principalmente por ser saudável e nutritiva. Quando não a consomem é porque não gostam - $33(39,3 \%)$ no sexo feminino - e $32(47,1 \%)$ no sexo masculino não sentem fome. Prevaleceu o percentual de $71,4 \%(n=55)$ no sexo feminino e $69,8 \%(n=30)$ no sexo

Tabela I - Descrição dos estudantes em relação ao estado nutricional pelo índice de massa corporal (IMC) e circunferência de cintura (CC), segundo o sexo. Lajeado-RS, 2013.

\begin{tabular}{|c|c|c|c|c|c|c|}
\hline \multirow{3}{*}{ Variável } & \multirow{3}{*}{ Categoria } & \multicolumn{4}{|c|}{ Sexo } & \multirow{3}{*}{$p^{*}$} \\
\hline & & \multicolumn{2}{|c|}{ Feminino } & \multicolumn{2}{|c|}{ Masculino } & \\
\hline & & $\mathrm{n}$ & $\%$ & $\mathrm{n}$ & $\%$ & \\
\hline \multirow{4}{*}{ Classificação IMC } & Baixo peso & 2 & 2,6 & - & - & 0,469 \\
\hline & Eutrofia & 56 & 72,7 & 28 & 65,1 & \\
\hline & Sobrepeso & 12 & 15,6 & 8 & 18,6 & \\
\hline & Obesidade & 7 & 9,1 & 7 & 16,3 & \\
\hline \multirow{2}{*}{ Classificação CC } & Adequado & 75 & 97,4 & 43 & 100,0 & 0,536 \\
\hline & Acima do adequado & 2 & 2,6 & - & - & \\
\hline
\end{tabular}

IMC: Índice de Massa Corporal; CC: Circunferência da Cintura.*Teste Exato de Fisher. 
masculino referindo achar "ótima ou boa" a alimentação oferecida pela escola, demonstrando uma boa aceitação.

Com relação à adesão, os resultados também foram positivos, já que a maioria dos estudantes do sexo feminino $(\mathrm{n}=44 ; 57,1 \%)$ e masculino $(\mathrm{n}=19 ; 44,2 \%)$ consome a alimentação escolar de duas a três vezes por semana (Tabela II).

A maioria dos estudantes do sexo feminino $(\mathrm{n}=55$; $71,4 \%)$ e do sexo masculino $(\mathrm{n}=24 ; 55,8 \%)$ diz trazer de casa bolachas, salgadinhos ou sanduíche com pão branco. Quanto ao que compram na escola, o sexo feminino prefere refrigerante, doces e guloseimas $(n=50 ; 64,9 \%)$, enquanto o sexo masculino prefere cachorro quente $(\mathrm{n}=21 ; 48,8 \%)$. Tanto o sexo feminino $(\mathrm{n}=49 ; 63,7 \%)$ quanto o masculino $(\mathrm{n}=30 ; 69,8 \%)$ geralmente tomam algum líquido com os alimentos (Tabela III).

Tanto os estudantes eutróficos $(\mathrm{n}=82 ; 97,6 \%)$ como os com sobrepeso/obesidade $(n=34 ; 100 \%)$ consideraram a alimentação escolar importante. Os eutróficos $(n=44 ; 52,4 \%)$ e os com sobrepeso/obesidade ( $\mathrm{n}=17 ; 50 \%)$ consumiam-na de 2 a 3 vezes na semana, e se os eutróficos $(n=33 ; 39,3 \%)$ não a consumiam é porque não gostavam da alimentação oferecida, enquanto os estudantes com sobrepeso/obesidade $(\mathrm{n}=16 ; 47,1 \%)$ era porque não tinham apetite ou fome. A maioria dos estudantes eutróficos $(n=57 ; 67,8 \%)$ e com sobrepeso/obesidade $(\mathrm{n}=26 ; 76,5 \%)$ considerou boas ou ótimas as preparações oferecidas na escola (Tabela IV).

Estudantes eutróficos - $57(67,9 \%)$ e $53(63,1 \%)$ - e com sobrepeso/obesidade - $21(61,8 \%)$ e $25(73,5 \%)$ trazem de casa, na maioria das vezes, bolachas, salgadinhos ou sanduíche com pão branco e tomam líquido junto com a alimentação. Independentemente do seu estado nutricional, a maioria dos estudantes eutróficos $(n=44 ; 52,4 \%)$ e dos portadores de sobrepeso/obesidade $(n=16 ; 47,1 \%)$ compra refrigerantes, doces, guloseimas, biscoitos doces ou bolos na escola (Tabela V).

Tabela II - Descrição dos estudantes em relação à alimentação escolar, segundo o sexo. Lajeado-RS, 2013.

\begin{tabular}{|c|c|c|c|c|c|c|}
\hline \multirow{3}{*}{ Variável } & \multirow{3}{*}{ Categoria } & \multicolumn{4}{|c|}{ Sexo } & \multirow{3}{*}{$p^{*}$} \\
\hline & & \multicolumn{2}{|c|}{ Feminino } & \multicolumn{2}{|c|}{ Masculino } & \\
\hline & & $\mathbf{n}$ & $\%$ & $\mathbf{n}$ & $\%$ & \\
\hline \multirow{2}{*}{$\begin{array}{l}\text { Acha importante a alimentação escolar } \\
\text { gratuita? }\end{array}$} & Sim & 77 & 100,0 & 41 & 95,3 & 0,126 \\
\hline & Não & - & - & 2 & 4,7 & \\
\hline \multirow{4}{*}{ Por que você acha importante? } & Saudável/Nutritiva & 46 & 59,7 & 23 & 53,5 & 0,539 \\
\hline & Saborosa/Gosto bom & 7 & 9,1 & 8 & 18,6 & \\
\hline & Sente fome na escola & 21 & 27,3 & 11 & 25,6 & \\
\hline & Outros & 3 & 3,9 & 1 & 2,3 & \\
\hline \multirow{4}{*}{$\begin{array}{l}\text { Quando você não come a alimentação } \\
\text { escolar gratuita, é por quê? }\end{array}$} & Sem apetite & 26 & 33,8 & 22 & 51,2 & 0,028 \\
\hline & Leva merenda de casa & 10 & 13,0 & 4 & 9,3 & \\
\hline & Compra na escola & 5 & 6,5 & 7 & 16,3 & \\
\hline & $\begin{array}{l}\text { Não gosta da } \\
\text { alimentação da escola }\end{array}$ & 36 & 46,8 & 10 & 23,3 & \\
\hline \multirow{3}{*}{$\begin{array}{l}\text { Quantas vezes por semana você come a } \\
\text { alimentação escolar gratuita? }\end{array}$} & 5 - 4 vezes & 20 & 26,0 & 12 & 27,9 & 0,263 \\
\hline & $3-2$ vezes & 44 & 57,1 & 19 & 44,2 & \\
\hline & $1-0$ vezes & 13 & 16,9 & 12 & 27,9 & \\
\hline \multirow{4}{*}{$\begin{array}{l}\text { Como você vê a alimentação escolar } \\
\text { gratuita preparada na escola? }\end{array}$} & Ótima & 14 & 18,2 & 16 & 37,2 & 0,063 \\
\hline & Boa & 41 & 53,2 & 14 & 32,6 & \\
\hline & Razoável & 18 & 23,4 & 12 & 27,9 & \\
\hline & Ruim & 4 & 5,2 & 1 & 2,3 & \\
\hline
\end{tabular}

*Teste Exato de Fisher. 
Tabela III - Descrição dos estudantes em relação à alimentação comprada nas cantinas escolares, à alimentação trazida de casa e aos líquidos consumidos, segundo o sexo. Lajeado-RS, 2013.

\begin{tabular}{|c|c|c|c|c|c|c|}
\hline \multirow{3}{*}{ Variável } & \multirow{3}{*}{ Categoria } & \multicolumn{4}{|c|}{ Sexo } & \multirow{3}{*}{$p^{*}$} \\
\hline & & \multicolumn{2}{|c|}{ Feminino } & \multicolumn{2}{|c|}{ Masculino } & \\
\hline & & $\mathbf{n}$ & $\%$ & $\mathbf{n}$ & $\%$ & \\
\hline \multirow{4}{*}{ O que você compra na cantina da escola? } & Nada/Nunca compro & 10 & 13,0 & 12 & 27,9 & $<0,001$ \\
\hline & Refrigerante, doces, guloseimas, & & & & & \\
\hline & biscoitos doces, bolos & 50 & 64,9 & 10 & 23,3 & \\
\hline & Cachorro quente & 17 & 22,1 & 21 & 48,8 & \\
\hline \multirow{3}{*}{$\begin{array}{l}\text { O que você traz, na maioria das vezes, de } \\
\text { casa para comer na escola? }\end{array}$} & $\begin{array}{l}\text { Bolachas, salgadinhos, sanduíche } \\
\text { não branco (carboidratos) }\end{array}$ & 55 & & & 558 & (18 \\
\hline & Frutas & $\begin{array}{c}55 \\
7\end{array}$ & $\begin{array}{l}71,4 \\
9,1\end{array}$ & $\begin{array}{c}24 \\
8\end{array}$ & $\begin{array}{l}3,0 \\
18,6\end{array}$ & 0,180 \\
\hline & Nunca traz nada de casa & 15 & 19,5 & 11 & 25,6 & \\
\hline \multirow{3}{*}{$\begin{array}{l}\mathrm{Na} \text { escola, você toma algum líquido } \\
\text { junto com os alimentos? }\end{array}$} & $\mathrm{Si}$ & 13 & 16,9 & 16 & 37,2 & 0,047 \\
\hline & Não & 28 & 36,4 & 13 & 30,2 & \\
\hline & Ás vezes & 36 & 46,8 & 14 & 32,6 & \\
\hline \multirow{5}{*}{ O que você costuma beber na escola? } & Água & 54 & 70,1 & 31 & 72,1 & 0,827 \\
\hline & Suco & 10 & 13,0 & 3 & 7,0 & \\
\hline & Leite com achocolatado & 4 & 5,2 & 3 & 7,0 & \\
\hline & Refrigerante & 8 & 10,4 & 6 & 14,0 & \\
\hline & Chá & 1 & 1,3 & - & 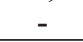 & \\
\hline
\end{tabular}

*Teste Exato de Fisher.

Tabela IV - Descrição dos estudantes em relação à alimentação escolar, segundo estado nutricional. Lajeado-RS, 2013.

\begin{tabular}{|c|c|c|c|c|c|c|}
\hline \multirow{3}{*}{ Variável } & \multirow{3}{*}{ Categoria } & \multicolumn{4}{|c|}{ Classificação IMC } & \multirow{3}{*}{$p^{*}$} \\
\hline & & \multicolumn{2}{|c|}{ Eutrofia } & \multicolumn{2}{|c|}{$\begin{array}{l}\text { Sobrepeso/ } \\
\text { Obesidade }\end{array}$} & \\
\hline & & $\mathbf{n}$ & $\%$ & $\mathbf{n}$ & $\%$ & \\
\hline \multirow{2}{*}{$\begin{array}{l}\text { Acha importante a alimentação escolar } \\
\text { gratuita? }\end{array}$} & $\operatorname{Sim}$ & 82 & 97,6 & 34 & 100,0 & 1,000 \\
\hline & Não & 2 & 2,4 & 0 & 0,0 & \\
\hline \multirow{4}{*}{ Por que você acha importante? } & Saudável/Nutritiva & 44 & 52,4 & 24 & 70,6 & 0,028 \\
\hline & Saborosa/gosto bom & 9 & 10,7 & 6 & 17,6 & \\
\hline & Sente fome na escola & 28 & 33,3 & 3 & 8,8 & \\
\hline & Outros & 3 & 3,6 & 1 & 2,9 & \\
\hline \multirow{4}{*}{$\begin{array}{l}\text { Quando você não come a alimentação } \\
\text { escolar gratuita, é por quê? }\end{array}$} & Sem apetite/sem fome & 32 & 38,1 & 16 & 47,1 & 0,386 \\
\hline & Leva merenda de casa & 12 & 14,3 & 2 & 5,9 & \\
\hline & Compra na escola & 7 & 8,3 & 5 & 14,7 & \\
\hline & Não gosta da merenda da escola & 33 & 39,3 & 11 & 32,4 & \\
\hline \multirow{3}{*}{$\begin{array}{l}\text { Quantas vezes por semana você come } \\
\text { a alimentação escolar gratuita? }\end{array}$} & 5 - 4 vezes & 23 & 27,4 & 9 & 26,5 & 0,928 \\
\hline & $3-2$ vezes & 44 & 52,4 & 17 & 50,0 & \\
\hline & $1-0$ vezes & 17 & 20,2 & 8 & 23,5 & \\
\hline \multirow{4}{*}{$\begin{array}{l}\text { Como você vê a alimentação escolar } \\
\text { gratuita preparada na escola? }\end{array}$} & Ótima & 20 & 23,8 & 10 & 29,4 & 0,569 \\
\hline & Boa & 37 & 44,0 & 16 & 47,1 & \\
\hline & Razoável & 22 & 26,2 & 8 & 23,5 & \\
\hline & Ruim & 5 & 6,0 & 0 & 0,0 & \\
\hline
\end{tabular}

IMC: Índice de Massa Corporal *Teste Exato de Fisher. 
Tabela V - Descrição dos estudantes em relação à alimentação comprada nas cantinas escolares, à alimentação trazida de casa e aos líquidos consumidos, segundo estado nutricional. Lajeado-RS, 2013.

\begin{tabular}{|c|c|c|c|c|c|c|}
\hline \multirow{3}{*}{ Variável } & \multirow{3}{*}{ Categoria } & \multicolumn{4}{|c|}{ Classificação IMC } & \multirow{3}{*}{$p^{*}$} \\
\hline & & \multicolumn{2}{|c|}{ Eutrofia } & \multicolumn{2}{|c|}{$\begin{array}{l}\text { Sobrepeso/ } \\
\text { Obesidade }\end{array}$} & \\
\hline & & n & $\%$ & n & $\%$ & \\
\hline \multirow{3}{*}{$\begin{array}{l}\text { O que você compra na cantina da } \\
\text { escola? }\end{array}$} & Nada/Nunca compro & 15 & 17,9 & 6 & 17,6 & 0,860 \\
\hline & $\begin{array}{l}\text { Refrigerante, doces, guloseimas, } \\
\text { biscoitos doces, bolos }\end{array}$ & 44 & 52,4 & 16 & 47.1 & \\
\hline & Cachorro quente & 25 & 29,8 & 12 & 35,3 & \\
\hline \multirow{3}{*}{$\begin{array}{l}\text { O que você traz, na maioria das } \\
\text { vezes, de casa para comer na escola? }\end{array}$} & $\begin{array}{l}\text { Bolachas, salgadinhos, } \\
\text { sanduíche pão branco }\end{array}$ & 57 & 67,9 & 21 & 61,8 & 0,679 \\
\hline & Frutas & 11 & 13,1 & 4 & 11,8 & \\
\hline & Nunca traz nada de casa & 16 & 19,0 & 9 & 26,5 & \\
\hline \multirow{3}{*}{$\begin{array}{l}\text { Na escola, você toma algum líquido } \\
\text { junto com os alimentos? }\end{array}$} & Sim & 22 & 26,2 & 7 & 20,6 & 0,296 \\
\hline & Não & 31 & 36,9 & 9 & 26,5 & \\
\hline & Ás vezes & 31 & 36,9 & 18 & 52,9 & \\
\hline \multirow{5}{*}{ O que você costuma beber na escola? } & Água & 64 & 76,2 & 20 & 58,8 & 0,113 \\
\hline & Suco & 8 & 9,5 & 4 & 11,8 & \\
\hline & Leite com achocolatado & 5 & 6,0 & 2 & 5,9 & \\
\hline & Refrigerante & 6 & 7,1 & 8 & 23,5 & \\
\hline & Chá & 1 & 1,2 & 0 & 0,0 & \\
\hline
\end{tabular}

IMC: Índice de Massa Corporal. *Teste Exato de Fisher.

\section{DISCUSSÃO}

O presente estudo demonstrou uma boa aceitação da alimentação escolar oferecida gratuitamente na escola e um alto consumo de alimentos calóricos quando os estudantes trazem os alimentos de casa ou os adquirem na cantina.

Quanto ao estado nutricional, verificou-se que $15,6 \%$ e $18,6 \%$ do sexo feminino e masculino, respectivamente, apresentaram sobrepeso. Resultados superiores a um estudo $^{(4)}$, no qual $11,4 \%$ dos alunos apresentaram sobrepeso. Outra pesquisa ${ }^{(14)}$ encontrou $9,5 \%$ de sobrepeso, enquanto outra investigação ${ }^{(15)}$ obteve $13,1 \%$.

Observou-se no presente estudo que a maioria dos estudantes estava com a medida da CC adequada, resultado que aponta menor risco de desenvolvimento de doenças cardiovasculares e síndrome metabólica na faixa etária infantil. A CC está relacionada ao excesso de gordura abdominal e a fatores de risco para doenças cardiovasculares, como colesterol total e LDL colesterol aumentados e HDL colesterol baixo ${ }^{(16)}$. A CC é um indicador de gordura visceral bem explorada na população adulta, mais recentemente identificada como um fator de risco em crianças e adolescentes ${ }^{(17)}$.
Apesar de a maioria dos estudantes se apresentar eutrófica na presente pesquisa, os percentuais de excesso de peso foram relevantes. A maioria dos eutróficos afirmou achar importante consumir a alimentação escolar porque sentem fome; e os com sobrepeso/obesidade, por ser saudável e nutritiva. Resultados que se contrapõem aos de um estudo ${ }^{(18)}$ segundo o qual os estudantes com excesso de peso sentem mais fome do que os eutróficos e, assim, alimentam-se mais vezes durante o dia, tendo o hábito de se alimentar entre as principais refeições.

Também se observou que, nas cantinas, o sexo feminino compra mais doces e refrigerantes; e o sexo masculino, mais cachorro quente $(p=<0,001)$. Esses resultados sobre os hábitos do consumo alimentar nas escolas são preocupantes, pois a carga de doenças crônicas está atingindo índices preocupantes para a saúde pública e, com isso, doenças como a obesidade e a hipertensão arterial atingem também crianças e adolescentes de forma semelhante à população $\operatorname{adulta}^{(19)}$.

No presente estudo, $10,4 \%$ do sexo feminino e $14 \%$ do sexo masculino relataram o consumo de refrigerante junto com a alimentação escolar, resultado inferior ao encontrado em outra pesquisa ${ }^{(20)}$, na qual $29 \%$ dos alunos (maioria do 
sexo feminino) tomavam refrigerante durante as refeições, enquanto outro estudo ${ }^{(21)}$ observou um maior consumo de refrigerante durante almoço e lanches (55\%).

A maioria dos estudantes questionados respondeu que consome alimentação escolar gratuita de 2 a 3 vezes por semana. Resultado semelhante a um estudo ${ }^{(22)}$ que encontrou um público de $58 \%$ de estudantes que às vezes consome a alimentação escolar. Ao mesmo tempo, no atual estudo, $16,9 \%$ do sexo feminino e $27,9 \%$ do sexo masculino responderam consumir a alimentação escolar no máximo uma vez por semana, resultados superiores ao de outra pesquisa $^{(23)}$, que observou que $7,7 \%$ dos estudantes nunca consumiam a alimentação escolar. Em outro estudo ${ }^{(8)}$, $61,1 \%$ relataram consumir de 4 a 5 vezes na semana a alimentação oferecida, resultados superiores ao do atual estudo. Em outros ${ }^{(7,23)}$, a maioria dos estudantes declarara consumir todos os dias.

No atual estudo, observou-se que as respostas dos estudantes sobre a aceitabilidade da alimentação escolar foram "boa" e "ótima" em sua maioria, resultado semelhante a outros estudos ${ }^{(21,22)}$, que encontraram $74 \%$ e $70,8 \%$, respectivamente, de boa aceitabilidade da alimentação escolar. Entretanto, esses percentuais são inferiores ao estabelecido pelo Programa Nacional de Alimentação Escolar para a aceitabilidade da alimentação escolar, que deve ser de, no mínimo, $85 \%$ para a escala hedônica ${ }^{(1)}$.

Observou-se na pesquisa em questão uma diferença significativa entre os sexos em relação ao motivo pelo qual não consumiam a alimentação oferecida na escola, pois o sexo feminino relatou que quando não consome é porque não gosta do que é oferecido e o sexo masculino, porque não sente fome, corroborando com outro estudo ${ }^{(24)}$, em que os entrevistados referiram não gostar da alimentação servida (50\%) ou traziam lanche de casa (25\%).

Quando não consomem a refeição disponibilizada gratuitamente, a maioria dos estudantes investigados no atual estudo mencionou trazer de casa bolachas, salgadinhos ou sanduíche com pão branco, mostrando que as preferências são semelhantes entre os sexos e as classificações de estado nutricional. Resultado semelhante a um estudo ${ }^{(24)}$, no qual $52,2 \%$ dos estudantes traziam de casa salgadinho tipo chips, biscoito recheado, suco e refrigerante, sendo também semelhante a outra pesquisa ${ }^{(25)}$, na qual $50,2 \%$ dos estudantes traziam de casa bolachas ou pipoca, evidenciando resultados preocupantes, uma vez que a preferência recai sobre alimentos de baixa qualidade nutricional.

Como limitação do presente estudo, destaca-se a utilização de um questionário estruturado para a avaliação de ingestão da alimentação escolar dos estudantes, além de o número amostral ter sido prejudicado pela não autorização dos responsáveis.
Por fim, sugerem-se medidas para promoção da saúde com estratégias de orientação nutricional sobre a qualidade dos alimentos para os estudantes em geral.

\section{CONCLUSÃO}

Concluiu-se que os estudantes eutróficos, com sobrepeso e obesidade, de ambos os sexos, consumiam de 2 a 3 refeições gratuitas oferecidas na escola por semana e demonstraram preferência em trazer de casa bolachas, salgadinhos e sanduíches com pão branco. Quando compravam seu alimento na escola, tanto os eutróficos como os em sobrepeso e obesidade preferiam refrigerantes, doces, guloseimas, doces e bolos. Já em relação ao sexo, o feminino preferia refrigerantes, doces, guloseimas, doces e bolos, enquanto o masculino preferia cachorro quente.

\section{REFERÊNCIAS}

1. Fundo Nacional de Desenvolvimento da Educação. Alimentação escolar [Internet]. [acesso em 2013 Abr 13]. Disponível em: http://www.fnde.gov.br/ programas/alimentacao-escolar/alimentacao-escolarapresentacao

2. Sobral F, Costa VMHM. Programa Nacional de Alimentação Escolar: Sistematização e Importância. Alim Nutr Araraquara. 2008;19(1):73-81.

3. Gabriel CG, Marino MC, Malchioretto OR, Vasconcelos FAG. Proposta e aplicabilidade de modelo para avaliação da gestão municipal do Programa Nacional de Alimentação Escolar. Cad Saúde Pública. 2014;30(8):1731-44.

4. Lucero LM, Piovesan CB, Fernandes DD, Ghisleni DR, Colpo E. Acompanhamento nutricional de crianças de baixa renda que se beneficiam do programa nacional de alimentação escolar (PNAE). Rev AMRIGS. 2010;54(2):156-61.

5. Bertin RL, Malkowsi J, Zutter LC, Ulbrich AZ. Estado nutricional, hábitos alimentares e conhecimento de nutrição em escolares. Rev Paul Pediatr. 2010;28(3):303-8.

6. Silva LAS. O fazer educação alimentar e nutricional: algumas contribuições para reflexão. Ciênc Saúde Coletiva. 2012;17(2):453-62.

7. Mendes ACL, Queiroz DJM, Melo ANL, Sureira TM, Pequeno NPF. Food and nutritional profile of infants treated in basic health unit in Rio Grande do Norte. J Hum Growth Dev. 2014;24(1):16-23.

8. Danelon MAS, Danelon MS, Silva MV. Serviços de alimentação destinados ao público escolar: análise da 
convivência do Programa de Alimentação Escolar e das cantinas. Segur Aliment Nutr. 2006;13(1):85-94.

9. Vitolo MR. Nutrição da gestação ao envelhecimento. Rubio: Rio de Janeiro; 2008.

10. Teixeira AB, Campos ALA, Paula RAC. A merenda escolar em Linhares: caminhos e descaminhos. Pesquisa Debate. 2009; Num esp:2-27.

11. Mota CH, Mastroeni SSS, Mastroeni MF. Consumo da refeição escolar na rede pública municipal de ensino. Rev Bras Est Pedag. 2013, 94(236):168-84.

12. Dal Bosco SM. Terapia Nutricional em Pediatria. São Paulo: 2010. Atheneu.

13. Organização Mundial da Saúde. Curvas 2007 [acesso em 2013 Nov 7]. Disponível em: http://nutricao.saude. gov.br/docs/geral/curvas_oms_2006_2007.pdf

14. Leal VS, Lira PIC, Oliveira JS, Menezes ECR, Souza AL, Arruda Neto MA, et al. Excesso de peso em crianças e adolescentes no Estado de Pernambuco, Brasil: prevalência e determinantes. Cad Saúde Pública. 2012;28(6):1175-1182.

15. Mazaro IAR, Zanolli ML, Morcilo MA, Zambon MP. Obesidade e fatores de risco cardiovascular em estudantes de Sorocaba, SP. Rev Assoc Med Bras. 2011;57(6):660-6.

16. Burgos MS, Burgos LT, Camargo MD, Franke SI, Prá D, Silva AMV, Borges TS, Todende PF, Reckziegel MB, Reuter CP. Associação entre medidas antropométricas e fatores de risco cardiovascular em crianças e adolescentes. Arq Bras Cardiol. 2013;201 (4):288-296.

17. Schommer VA, Barbiero SM, Cesa CC, Silva AD, Pellanda LC. Excesso de Peso, Variáveis Antropométricas e Pressão Arterial em Escolares de 10 a 18 Anos. Arq Bras Cardiol. 2014;102(4):312-8.

18. Fagundes ALN, Ribeiro DC, Naspitz L, Garbelini LEB, Vieira JKP, Silva AP, et al. Prevalência de sobrepeso e obesidade em escolares da região de Parelheiros do município de São Paulo. Rev Paul Pediatr. 2008;26(3):212-7.

19. Ferreira JS, Aydos RD. Prevalência de hipertensão arterial em crianças e adolescentes obesos. Ciênc Saúde Coletiva. 2010;15(1):97-104.

20. Estima CCP, Philippi ST, Araki EL, Leal GVS, Martinez MF, Alvarenga MS. Consumo de bebidas e refrigerantes por adolescentes de uma escola pública. Rev Paul Pediatr. 2011;29(1):41-5.

21. Oliveira ACS, Santos EP, Silva MS, Vieira TPR, Silva SM. O impacto do consumo de refrigerantes na saúde de escolares do Colégio Gissoni. Rev Eletrônica Novo Enfoque. 2011;12(12):68-79.

22. Schwarz K, Munaretto LM, Castilho AJ, Kuhl AM. Aceitabilidade da merenda escolar oferecida em uma escola municipal. In: Anais do XVIII Encontro Anual de Iniciação Científica (EAIC); 2009 Set. 30 a Out. 02; Campus da Universidade Estadual de Londrina. Londrina: Universidade Estadual de Londrina; 2009. p. 1.

23. Teo CRPA, Corrêa EM, Gallina LS, Fransozi C. Programa nacional de alimentação escolar: adesão, aceitação e condições de distribuição de alimentação na escola. Nutrire Rev Soc Bras Aliment Nutr. 2009;34(3):165-85.

24. Santos AP, Pinheiro CT, Maciel FV, Geiger JM, Ramires MCC. Adesão ao Programa Nacional de Alimentação Escolar por estudantes de uma escola municipal de Pelotas, RS. In: Anais do XIX Congresso de Iniciação Científica (CIC), XII Encontro de Pós-Graduação (ENPOS) e II Mostra Científica, 2010 Nov 9, Pelotas/ RS [acesso em 2013 Nov 7]. Disponível em: http:// www2.ufpel.edu.br/cic/2010/cd/pdf/CS/CS_01164.pdf

25. Muniz VM, Carvalho AT. O Programa Nacional de Alimentação Escolar em município do estado da Paraíba: um estudo sob o olhar dos beneficiários do Programa. Rev Nutr. Campinas. 2007;20(3):295-6.

\section{Endereço primeiro autor:}

Jeani Tedeschi Ferreira

Centro Universitário Univates

Rua Avelino Tallini, 171

Bairro: Universitário

CEP: 95900-000 - Lajeado - RS - Brasil

E-mail: jeanitf@ibest.com.br

\section{Endereço de correspondência:}

Fernanda Scherer Adami

Centro Universitário Univates

Rua Avelino Tallini, 171

Bairro: Universitário

CEP: 95900-000 - Lajeado - RS - Brasil

E-mail: fernandascherer@univates.br 